\title{
Early diagnosis and management of pulmonary vein thrombosis following lung transplant
}

Federico Sertic, MD, ${ }^{a}$ Maria M. Crespo, MD, ${ }^{b}$ Bonnie Milas, MD, ${ }^{c}$ and Christian Bermudez, MD, ${ }^{a}$ Philadelphia, $\mathrm{Pa}$

From the ${ }^{\mathrm{a}}$ Division of Cardiovascular Surgery, Department of Surgery, ${ }^{\mathrm{b}}$ Division of Pulmonology, Department of Medicine, and ${ }^{\mathrm{c}}$ Department of Intensive Care, University of Pennsylvania, Perelman School of Medicine, Philadelphia, $\mathrm{Pa}$.

No source of funding is declared.

Disclosures: Authors have nothing to disclose with regard to commercial support.

Received for publication Sept 26, 2018; revisions received Jan 8, 2019; accepted for publication Feb 1, 2019; available ahead of print March 14, 2019.

Address for reprints: Federico Sertic, MD, 3400 Spruce St, Philadelphia, PA 19104 (E-mail: federico.sertic@ uphs.upenn.edu).

J Thorac Cardiovasc Surg 2019;157:e419-21

$0022-5223 / \$ 36.00$

Crown Copyright (c) 2019 Published by Elsevier Inc. on behalf of The American Association for Thoracic Surgery https://doi.org/10.1016/j.jtcvs.2019.02.002

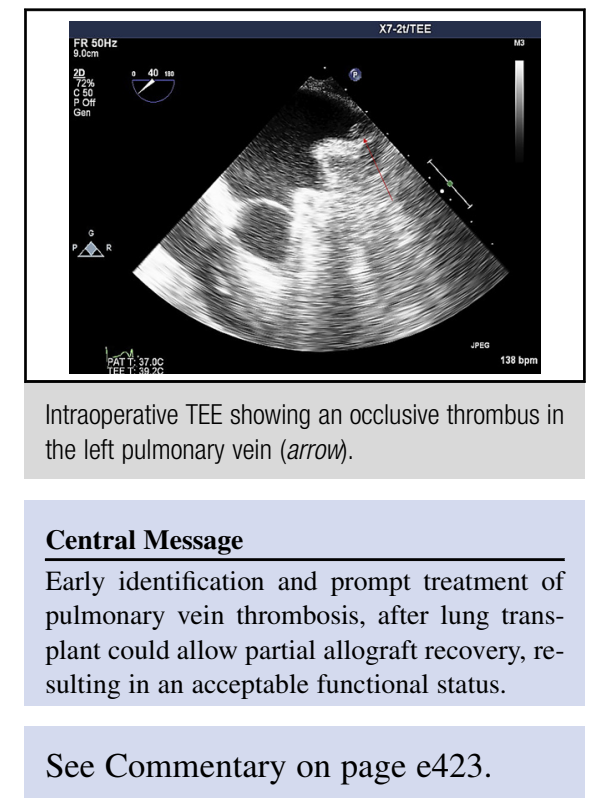

Pulmonary vein thrombosis (PVT) is a serious complication of lung transplantation that can lead to loss of the allograft and death of the recipient; however, limited information is available regarding its diagnosis and management, especially in patients with hemodynamically significant obstruction. ${ }^{1-5}$ We report the case of a lung transplant recipient supported by preoperative extracorporeal membrane oxygenation (ECMO) who had PVT develop soon after transplant.

\section{CLINICAL SUMMARY}

A 37-year-old woman with a history of pulmonary arterial hypertension and an atrial septostomy for symptomatic relief was listed for double-lung transplant. During her wait for an organ, she had a rapid decompensation and was placed on venoarterial ECMO support with cannulation of the right atrium and the left atrial appendage (Figure 1). Her chest was closed, and she was extubated and actively rehabilitated.

After 40 days of venoarterial ECMO support (Figure 2, $A$ ) with the chest closed, the patient underwent bilateral lung transplant, closure of her atrial septostomy, and repair of the ECMO cannulation sites on cardiopulmonary bypass (Figure 2, B). Because of her complex surgery, significant transfusions (10 units of packed red blood cells, 5 units of fresh-frozen plasma, 4 pf units platelets, and 10 units of cryoprecipitate) and the high-risk of primary graft dysfunction, the patient was transferred to the intensive care unit with

venoarterial ECMO support (right atrium to aorta) with her chest open. An intraoperative transesophageal echocardiographic (TEE) assessment of the vascular anastomosis was performed immediately after attempting to wean the patient from venoarterial ECMO and showed an intact anastomosis with no evidence of stenosis or high velocity (right pulmonary vein [PV], $72.3 \mathrm{~cm} / \mathrm{s}$; left PV, $46.4 \mathrm{~cm} / \mathrm{s}$ ). The patient presented soon after with hypotension necessitating reinstitution of full ECMO flows, and we were unable to perform a complete and definite perioperative PV evaluation. The PV anastomosis were performed with a running 4-0 Prolene (Ethicon Inc, Somerville, NJ) suture taking extreme care to avoid including any muscle in the suture line or the atrial lumen. Early chest reexploration and evacuation of a left hemothorax was necessary for postoperative bleeding. Intravenous heparin infusion was started 12 hours after chest reexploration. The patient then remained in stable condition until postoperative day 2 , when she presented with left unilateral pulmonary edema. A chest radiograph showed complete opacification of the left side of the chest (Figure 2,D). No blood was found in the left side of the chest, but a TEE revealed no flow in the left PV, and the anastomosis appeared mildly stenotic at the suture line, with an occlusive thrombus (Figure 2,E). We placed the patient on cardiopulmonary bypass (with full heparinization), clamped the ascending aorta, opened the left atrium at the 


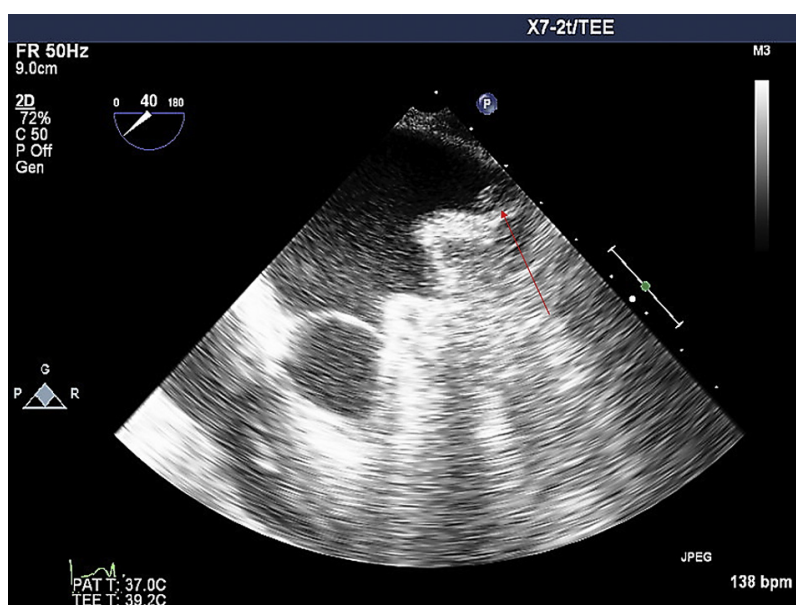

FIGURE 1. Intraoperative view of extracorporeal membrane oxygenation cannulation before bilateral lung transplant. The patient was put on central extracorporeal membrane oxygenation support, pulmonary artery (on the right) to left atrial appendage (on the left) at a local institution because of acute decompensation. The chest was closed to allow extubation and active rehabilitation during the wait for organ availability. suture line, and removed a $2 \times 3-\mathrm{cm}$ thrombus. The left atrium was directly opened without clamp placement to avoid dislodging the thrombus, the left PV anastomosis was then reconstructed with a running 4-0 Prolene suture. Total cardiopulmonary bypass time and crossclamp time were 85 and 42 minutes, respectively.

After surgery, the patient returned to the intensive care unit on venoarterial ECMO support. Inotropes and vasopressors were gradually tapered, and after 4 days, she underwent uneventful ECMO decannulation and chest closure, with adequate TEE assessment of the PV anastomosis. She was maintained on a low-level anticoagulation regimen (partial thromboplastin time, 50-60 seconds) for 2 weeks and discharged to a rehabilitation facility on postoperative day 23 on a long-term anticoagulation regimen with warfarin.

At her 6-month posttransplant follow-up, the patient displayed important functional recovery, with no clinical limitations. Forced expiratory volume in 1 second was $70 \%$ of predicted, forced vital capacity was $62 \%$ of predicted, and the ratio of the two was $93 \%$. A ventilation-perfusion scan
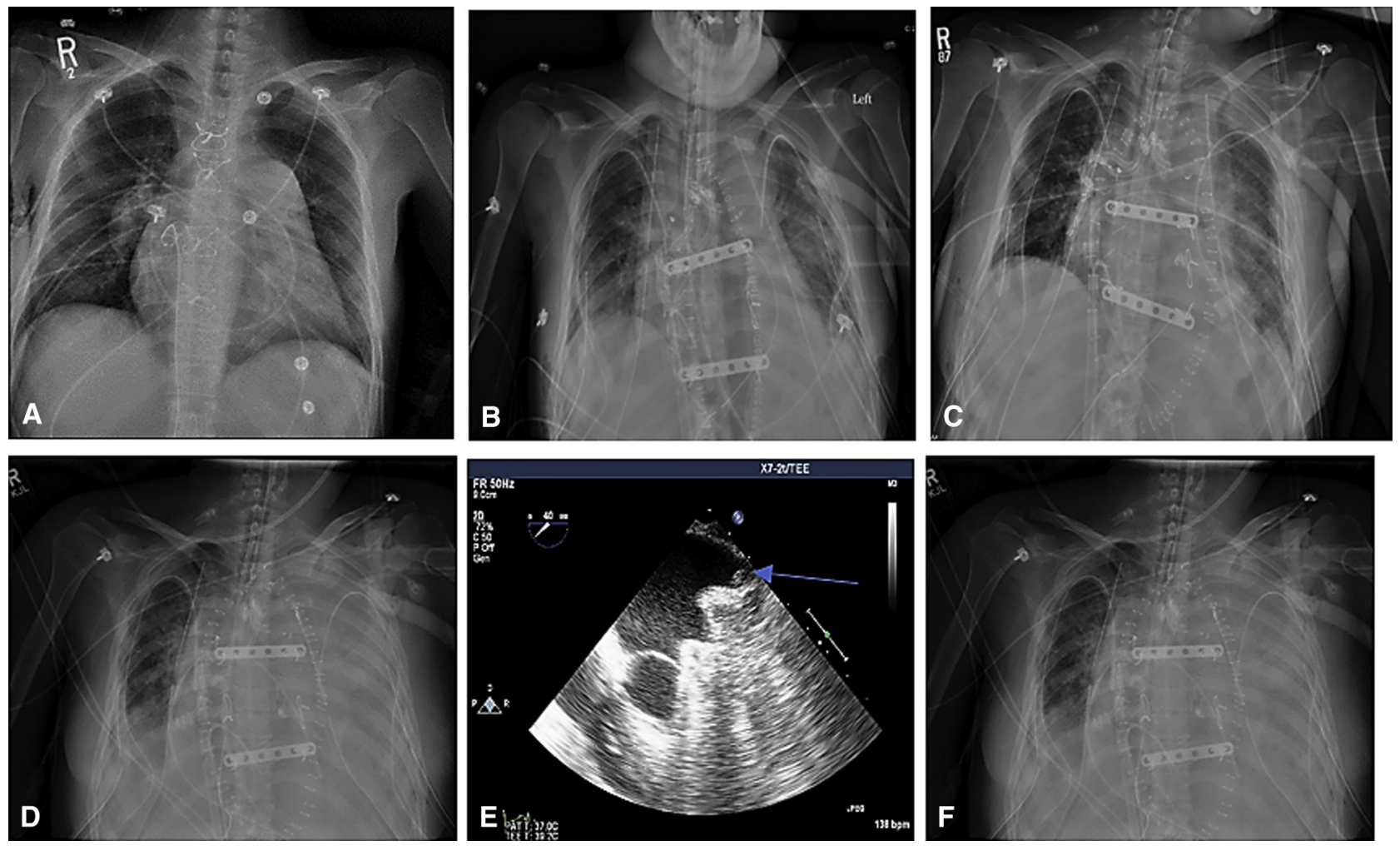

FIGURE 2. Chest imaging during hospital stay. A, On arrival to our institution, before transplant. Note the extracorporeal membrane oxygenation cannulation sites: right atrium to left atrial appendage. B, Immediately after bilateral lung transplant: open chest with central extracorporeal membrane oxygenation cannulation, right atrium to ascending aorta. C, Postoperative day 1 after evacuation of left-sided hemothorax. D, Postoperative day 2. Note the complete white out of the left lung, raising concerns for pulmonary vein thrombosis. E, Intraoperative transesophageal echocardiogram showing an occlusive thrombus with absence of flow in the left pulmonary vein (arrow). F, Immediately after the PV anastomosis was refashioned: open chest with central extracorporeal membrane oxygenation cannulation. 

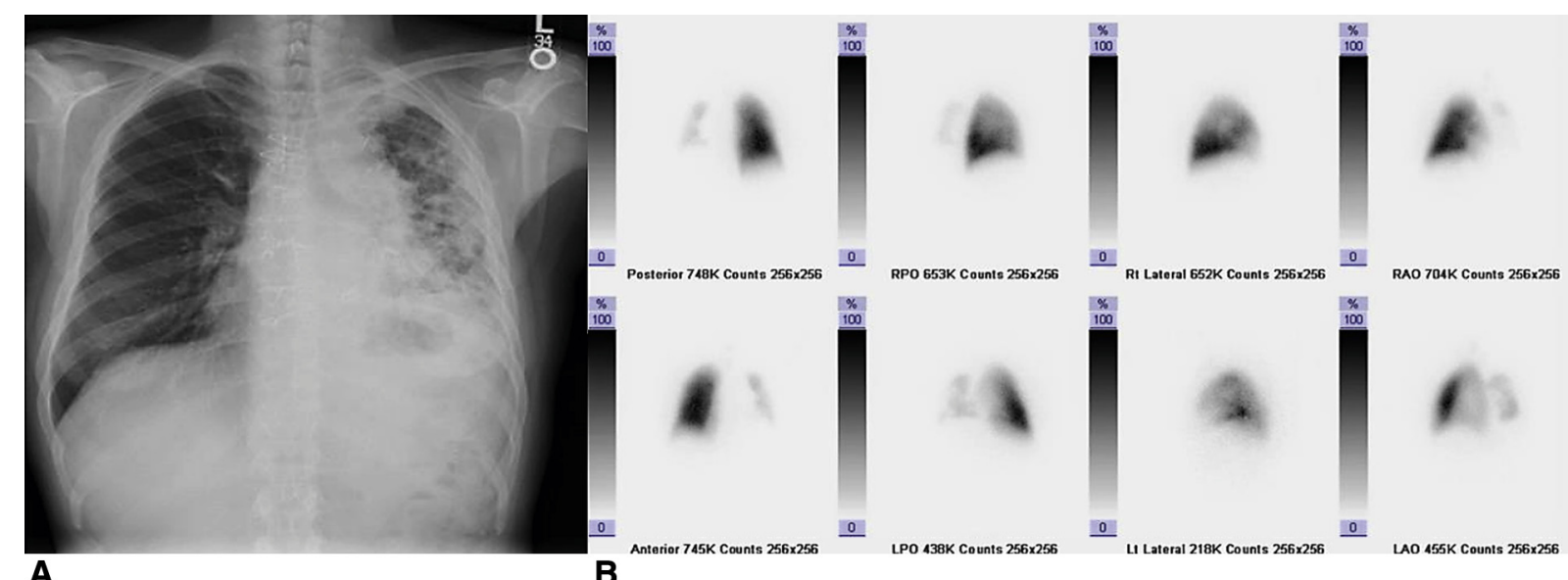

FIGURE 3. Imaging and lung function during follow-up. A, Chest radiograph on postoperative day 60 , showing partial recovery of the left lung with interstitial fibrosis and clear right lung field. B, Ventilation-perfusion scan at follow-up (6 months), showing $10 \%$ perfusion of the left lung and good right lung perfusion.

at 6 months showed partial recovery (10\% perfusion) of the left lung (Figure 3).

\section{DISCUSSION}

PVT is a feared and potentially fatal complication of lung transplantation. In this report, we highlight the importance of early suspicion, early diagnosis, and prompt treatment of PVT after lung transplantation. The initial presentation of PVT is similar to those of more common posttransplant complications. ${ }^{1}$ A high index of suspicion should be maintained in patients with unilateral, dense x-ray infiltrates in the early postoperative period, especially when patients are kept on venoarterial ECMO support after transplant. ECMO can lead to a low-blood flow state in the pulmonary circulation, resulting in an increased risk of thrombotic

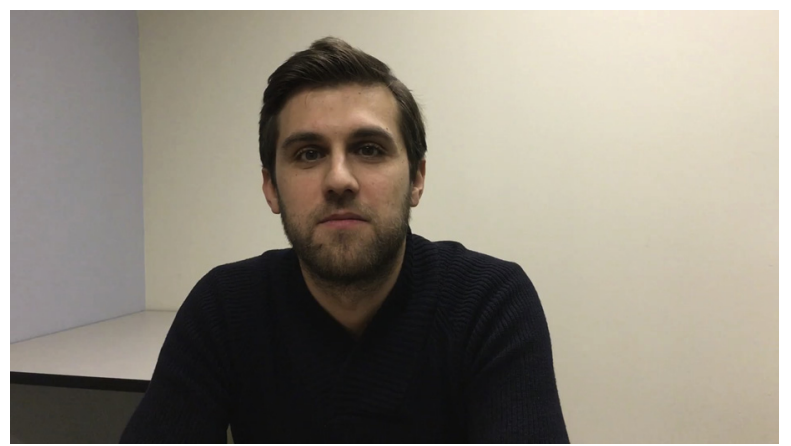

VIDEO 1. Dr Federico Sertic explaining key points of this report. Video available at: https://www.jtcvs.org/article/S0022-5223(19)30364-2/fulltext. complications, especially at the anastomotic sites. This situation should be managed with aggressive volume resuscitation, especially in a bleeding patient. TEE is a useful diagnostic tool; however, the effect of pulmonary artery unloading during venoarterial ECMO support could give misleading findings of difficult interpretation (Video 1). For occlusive thrombi, surgical thrombectomy or thrombolytic therapy are options only after early diagnosis. ${ }^{2-5}$ If the diagnosis of PVT is made too late, a second major surgery (pneumonectomy or retransplantation) may be necessary, and this is associated with high morbidity and mortality. In our case, early recognition with TEE and early surgical thrombectomy allowed a partial recovery of the affected lung, sparing the patient from pneumonectomy or retransplantation and resulting in an acceptable functional status and quality of life.

\section{References}

1. Leibowitz DW, Smith CR, Michler RE, Ginsburg M, Schulman LL, McGregor CC, et al. Incidence of pulmonary vein complication after lung transplantation: a prospective transesophageal echocardiographic study. J Am Coll Cardiol. 1994;24:671-5.

2. Shah AS, Michler RE, Downey RJ, Leibowitz DW, Homma S, Smith CR. Management strategies for pulmonary vein thrombosis following single lung transplantation. J Card Surg. 1995;10:169-78.

3. Pham SM, Armitage JM, Katz WE, Griffith BP. Left atrial thrombosis after lung transplantation. Ann Thorac Surg. 1995;59:513-5.

4. Sarsam MAI, Yonan NA, Beton D, McMaster D, Deiraniya AK. Early pulmonary vein thrombosis after single lung transplantation. J Heart Lung Transplant. 1993; 12:17-9.

5. Nagahiro I, Horton M, Wilson M, Bennetts J, Spratt P, Glanville AR. Pulmonary vein thrombosis treated successfully by thrombectomy after bilateral sequential lung transplantation: report of a case. Surg Today. 2003;33:282-4. 